\title{
Stereotactic body radiation therapy for liver tumours using flattening filter free beam: dosimetric and technical considerations
}

\author{
Pietro Mancosu ${ }^{1 *}$, Simona Castiglioni ${ }^{1}$, Giacomo Reggiori ${ }^{1}$, Maddalena Catalano ${ }^{1}$, Filippo Alongi ${ }^{1}$, Chiara Pellegrini ${ }^{1}$ \\ , Stefano Arcangeli ${ }^{1}$, Angelo Tozzi ${ }^{1}$, Francesca Lobefalo ${ }^{1}$, Antonella Fogliata ${ }^{2}$, Piera Navarria ${ }^{1}$, Luca Cozzi ${ }^{2}$ and \\ Marta Scorsetti ${ }^{1}$
}

\begin{abstract}
Purpose: To report the initial institute experience in terms of dosimetric and technical aspects in stereotactic body radiation therapy (SBRT) delivered using flattening filter free (FFF) beam in patients with liver lesions.

Methods and Materials: From October 2010 to September 2011, 55 consecutive patients with 73 primary or metastatic hepatic lesions were treated with SBRT on TrueBeam using FFF beam and RapidArc technique. Clinical target volume (CTV) was defined on multi-phase CT scans, PET/CT, MRI, and 4D-CT. Dose prescription was 75 Gy in 3 fractions to planning target volume (PTV). Constraints for organs at risk were: $700 \mathrm{cc}$ of liver free from the 15 Gy isodose, $\mathrm{D}_{\max }<21$ Gy for stomach and duodenum, $\mathrm{D}_{\max }<30 \mathrm{~Gy}$ for heart, $\mathrm{D}_{0.1} \mathrm{cc}<18$ Gy for spinal cord, $\mathrm{V}_{15}$ Gy $<$ $35 \%$ for kidneys. The dose was downscaled in cases of not full achievement of dose constraints. Daily cone beam $\mathrm{CT}(\mathrm{CBCT})$ was performed.

Results: Forty-three patients with a single lesion, nine with two lesions and three with three lesions were treated with this protocol. Target and organs at risk objectives were met for all patients. Mean delivery time was $2.8 \pm 1.0$ min. Pre-treatment plan verification resulted in a Gamma Agreement Index of $98.6 \pm 0.8 \%$. Mean on-line coregistration shift of the daily $C B C T$ to the simulation $C T$ were: $-0.08,0.05$ and $-0.02 \mathrm{~cm}$ with standard deviations of $0.33,0.39$ and $0.55 \mathrm{~cm}$ in, vertical, longitudinal and lateral directions respectively.
\end{abstract}

Conclusions: SBRT for liver targets delivered by means of FFF resulted to be feasible with short beam on time. Keywords: SBRT, liver, flattening filter free, RapidArc, TrueBeam

\section{Introduction}

Stereotactic body radiation therapy (SBRT) has proved its efficacy in several patient populations with primary and metastatic limited tumours [1]. In particular, SBRT may be appropriate for selected patients with oligometastatic disease, defined as less than five lesions [2] or with organ-confined limited volume primary tumours. Abdominal SBRT has been reported with reference mainly to primary and secondary liver tumours $[1,3,4]$. It is known that in the setting of limited tumour burden, SBRT leads to local control rates higher than $70 \%-80 \%$ [1], which may improve survival and quality of life.

\footnotetext{
* Correspondence: pietro.mancosu@humanitas.it

'IRCCS Istituto Clinico Humanitas, Rozzano (Milano), Italy

Full list of author information is available at the end of the article
}

RapidArc (RA) is a relative new VMAT technique based on simultaneous optimisation of multi leaf collimator (MLC) shapes, dose rate and gantry rotation speed [5]. The technology was investigated in several studies, showing comparable target coverage and a general improvement in organs at risk (OAR) and healthy tissue sparing, a reduced beam-on time and lower number of monitor units (MU) compared to other IMRT approaches [6-13]. In a previous work we demonstrated the feasibility and dosimetric advantage to use Volumetric Modulated Arc Therapy (VMAT) using RapidArc (RA) (Varian Medical Systems, Palo Alto, CA) in SBRT treatments of abdominal region to reduce treatment time, compared to 3D conformal radiotherapy (3DCRT) and intensity modulated radiotherapy (IMRT)

\section{C) Biomed Central}


techniques [14]. Thus since November 2008 SBRT to abdominal targets has been delivered by means of RA. More recently, we reported our early experience in terms of technical feasibility, local control rate and acute toxicity profile of SBRT with RA for patients with primary or secondary abdominal tumours, showing the good toxicity profile and clinical results $[15,16]$.

TrueBeam (Varian Medical System, Palo Alto, USA) is a new accelerator designed for delivering both in flattened filter (FF) and flattering filter free (FFF) modality [17]. In particular, the removal of flattening filter was shown to reduce of out-of-field dose due to the reduction of head scatter and residual electron contamination, consequence of the exclusion of beam attenuation due to flattened filter. Furthermore, with FFF beams the dose rate is increased up to a factor 4 for the $10 \mathrm{MV}$ beam [18-23]. This leads to a possible reduction in delivery time with benefit in patient discomfort and with potential limitation of intra-fraction motion.

Some feasibility studies for SBRT using FFF beams are present in literature $[23,24]$ and in a recent study performed in our institute we showed our early experience in the use of FFF beams for SBRT treatments including liver metastases, lung primitive and metastases, isolated abdominal lymph nodes, adrenal glands, and pancreas [25]. In the present study our attention was focused on the sub-group of patients that underwent a strongly hypo-fractionated treatment for which the time advantage is maximum. In particular our aim was to show a complete overview of SBRT liver protocol focusing on technical aspects: the deliverability is evaluated by gamma agreement index using two different devices (MatriXX and GafChromic), the analysis of the interfraction displacements by means of CBCT, and dosimetric objectives according to the number of lesions treated. The evaluation of radiation-induced liver disease (RILD), the radiobiological consequence due to the higher dose rate, and the clinical evaluation are not aim of this paper and these aspects will be evaluated in a specific study.

\section{Methods and materials}

\section{Patients selection and treatment planning}

Between November 2010 and September 2011 at Istituto Clinico Humanitas (ICH) 55 patients with 73 primary or metastatic liver tumours were treated by TrueBeam in an on-going phase II prospective protocol approved by the internal ethical committee. Aim of the protocol, approved in late 2009, was the evaluation of local control in patients with liver malignancies; secondary endpoints were the acute and late toxicities, and evaluation of overall survival. According with Fleming approach, to demonstrate local control of at least $80 \%$ with a power of $90 \%$, at least forty-four patients in three years were necessary to complete the study. At present, all the patients are recruited and the follow-up are ongoing therefore no analysis regarding local control or toxicities will be reported in the present study.

Of the patients evaluated, 11 had primary liver tumours, and 44 had hepatic metastases from colon (23), biliar duct-pancreas (6), breast (5), and other sites (10). All the patients had been considered unfit for surgery or other non-surgical treatments at the time of radiation. Chemotherapy was stopped at least 3 weeks before SBRT and withheld until disease progression. Median patient age was 63 years (range: 43-83). Only patients with at least $1000 \mathrm{~cm}^{3}$ of liver free from the disease and with $\leq$ than 3 lesions were considered eligible to the protocol.

Disease extension was evaluated in all cases by Computed Tomography $(\mathrm{CT})$ with contrast. Although they were not inclusion criteria of the study, magnetic resonance imaging (MRI) and positron emission tomography/CT using FDG tracer (PET/CT) were acquired in respectively 2 and 14 patients. Both MRI and PET/CT were acquired for those patients whose treatment indication for radiotherapy was justified by these diagnostic exams; thus PET and MRI were only an integration in the target definition since the use of PET and MRI in defining the tumour is under evaluation and will be topic of future work. Computed tomography scans for planning were acquired for all patients positioned supine with their arms above the head; patients were immobilized by means of a thermoplastic body mask including a Styrofoam block for abdominal compression to minimize internal organ motion. Contrast free and 3 phases contrast-enhanced planning $\mathrm{CT}$ scans were acquired in free quiet breathing mode at $3 \mathrm{~mm}$ slice thickness with a stereotactic body frame to localise the isocentre. Breath hold in simulation CT was not mandatory in the study as many patients were unfit to maintain breath hold for many seconds with the compressor. In case of collaborative patients the simulations CT were performed in voluntary exhale breath hold.

Furthermore in case of lesions located in the VII or VIII hepatic segment or in case of liver cupola shift greater than $5 \mathrm{~mm}$ on the four simulation CTs, a four dimensional CT (4D-CT) was performed to best define the target margin. In addition, in two cases presenting internal clips due to previous surgery, the 4D-CT scan was acquired and the SBRT was performed in gated modality with internal marker tracking by $2 \mathrm{D}$ imaging. This option was released in July 2011 with TrueBeam version 1.5, and thus the first patients were not treated with internal marker tracking. This topic will be deepened in a specific paper.

The gross tumor volume (GTV) included macroscopic disease defined on CT as well as on PET if available. 
The clinical target volume (CTV) was defined equal to the GTV. The planning target volume (PTV) was generated by taking into account both the internal margin (IM) and the set-up margin (SM). Since SM was maintained at a minimum by the cone-beam CT (CBCT) daily verification of set up variations, the overall CTVPTV margin was prescribed as $8-12 \mathrm{~mm}$ in the cranialcaudal axis and 4-6 $\mathrm{mm}$ in the anterior-posterior and lateral axes, allowing mainly for residual intra-fraction target motion as well as for inaccuracies in CBCT image interpretation [26,27]. The organs at risks (OAR) considered were: healthy liver, spinal cord, kidneys, stomach, duodenum, heart, small bowel, oesophagus and ribs, in relation with the lesion location.

The isodose distribution applied during SBRT typically includes planned heterogeneity within the tumor intended to intensify the dose within the tumor. In this protocol, the isodose line prescribed to cover the PTV was at least $67 \%$ of the prescribed dose (range 67-95\%), trying to maximizing it up to $95 \%$ [28]. Dose prescription was set to 75 Gy in 3 consecutive daily fractions. For OARs, plans were required to meet the following objectives: $\mathrm{V}_{15}$ Gy (volume receiving $15 \mathrm{~Gy}$ ) < (total liver volume $-700 \mathrm{~cm}^{3}$ ) for healthy liver, $D_{0.1 \mathrm{~cm} 3}$ for spinal cord $<18$ Gy (dose at a volume of $0.1 \mathrm{~cm}^{3}$ should be lower than 18 Gy), $V_{15 \text { Gy }}<35 \%$ for both kidneys, $V_{21}$ Gy $<1 \%$ for duodenum, small bowel, oesophagus, and stomach, $\mathrm{V}_{30 \text { Gy }}<1 \%$ for heart; $\mathrm{D}_{30} \mathrm{~cm}^{3}<30$ Gy for ribs were considered as a secondary objective [3,29]. In case of overlap between PTV and duodenum or stomach, the priority was given to the OAR cropping the PTV to comply with the OAR limits.

\section{Treatment delivery}

All plans were designed and optimised with RA technique using the optimizer PROIII for a Varian TrueBeam equipped with a Millennium multi-leaf collimator (MLC) with a leaf width of $5 \mathrm{~mm}$ at the isocentre. RA plans were designed using full (i.e. $360^{\circ}$ ) or partial (i.e. around $200^{\circ}$ ) multiple arcs according in order to achieve the best dose distributions. Specifically, partial arcs were used in cases of (1) lesion located far from the median axis (i.e. more than $10 \mathrm{~cm}$ ) to do not collide the gantry with the couch induced by laterality of the couch, and (2) lesion very close to serial OARs (i.e. heart, gastrointestinal organs) to best protect them. Where possible, coplanar arcs were employed to fasten the delivery time, otherwise, non coplanar arcs arrangements were used with two perpendicular couch positions. In particular, the non-coplanar approach was adopted in multi-lesions cases only. All dose distributions were computed with the Analytical Anisotropic Algorithm (AAA) (version 10.0.28) implemented in the Eclipse planning system with a calculation grid resolution of at maximum 2.0 $\mathrm{mm}$.

Treatment was delivered in 3 consecutive working days, with the patient keeping a 3-hour fast to avoid gross displacement of stomach. Treatment delivery included stereotactic frame localization in the first session aiming to a preliminary isocentre positioning followed by image guidance with on-line couch adjustment at each fraction by means of cone beam CT (CBCT). Couch repositioning was operated after automatic matching of CBCT images to reference planning CT, followed by manual refining. The shift values were analyzed for all patients and random $(\sigma)$ and systematic $(\Sigma)$ population errors were calculated according to Van Herk approach [30]. In two cases the delivery was performed in respiratory gated modality. This approach allows the radiation beam to be turned off when respiratory movements place the target outside of the predetermined positioning parameters, and to resume the radiation when the target falls back within the accepted alignment. In particular, the respiration path was revealed with RPM system (Varian) and internal markers, previously detected on the simulation CT. The markers were detected by instantaneous $\mathrm{kV}$-portal images acquired before each beam-on phase. On each of these $\mathrm{kV}$-portal images, a circular region of interest (ROI) of $5 \mathrm{~mm}$ radius defined the theoretical marker's position for each projection; the radiation oncologist could then verify the instantaneous marker's positions to be inside the ROI, highlighting possible internal organ motions.

Before treatment, each plan was verified to assess dosimetric agreement between computed and delivered dose distributions. This quality assurance process was performed with two independent quality assurance systems (MatriXX and Gafchromic). The results of these measurements were scored in terms of the Gamma Agreement Index (GAI) based on the $\gamma$ of Low analysis [31] with thresholds: Distance to Agreement $=3 \mathrm{~mm}$, $\Delta$ Dose $=3 \%$.

\section{Data analysis and statistics}

Technical parameters of delivery were scored in terms of number of arcs, total number of monitor units (MU), monitor units per Gy (MU/Gy), total beam on time, total treatment time (the time in which the patient is in the bunker), and isocenter shift. Dosimetric quality of treatments was measured on the basis of dose volume histogram (DVH) analysis. For CTV and PTV the following data were reported: target coverage (mean, $\mathrm{D}_{1 \%}$, $\mathrm{D}_{95 \%}, \mathrm{~V}_{95 \%}, \mathrm{~V}_{107 \%}$ ) and conformity for PTV. Conformity index $\left(\mathrm{CI}_{95 \%}\right)$ was defined as the ratio between the volume of patient irradiated at $95 \%$ of the prescribed 
dose and the target volume. For OARs, the mean dose, the maximum dose $\left(D_{\mathrm{xcm} 3}\right)$ and appropriate values of $\mathrm{V}_{\mathrm{xGy}}$ (volume receiving at least $\mathrm{x}$ Gy) were scored. The data were reported separately for patients with 1 lesion and with 2-3 lesions. The Kolmogorov-Smirnoff test was applied to the data to evaluate if the data were normally distributed.

\section{Results}

Forty-three patients with a single lesion, nine with two lesions and three with three lesions were treated in this protocol, for a total of 73 isocenters. Figure 1 shows examples of dose distributions for three representative patients. Similar results were obtained in all patients. PTV and organs at risk are outlined as solid lines in the images. Table 1 reports the summary of dosimetric results.

The Kolmogorov-Smirnoff test revealed the data were normal distributed, thus the mean and standard deviation were used to present the results. In seven patients (i.e. $12 \%$ of cases) a $20 \%$ dose downscaling was necessary to comply with the liver constraint $\left(\mathrm{V}_{15}\right.$ Gy free $>$ $700 \mathrm{~cm}^{3}$ ). In all cases the maximum dose resulted satisfactory with negligible PTV volumes exceeding $107 \%$. Planning objectives for organs at risk were largely met in most of the cases for both groups of patients. The always resulted $>700 \mathrm{cc}$ as requested by the constraints, with mean and SD values of $965 \pm 141 \mathrm{cc}$ and $1283 \pm 706 \mathrm{cc}$ for single and multi lesion patients respectively. The higher $\mathrm{V}_{15}$ Gy free liver value for multi lesion patients was due to the larger liver volumes of this cohort of patients; the high mean and SD values are ascribable to a single case in which the total liver volume was $3320 \mathrm{cc}$ (excluding the case, the mean value decreases to $1039 \pm 157 \mathrm{cc}$ ).

Pre-treatment quality assurance measurements resulted satisfactory with a minimum deviation between groups of less than $3 \%$ and no statistically significant differences $(98.7 \pm 1.1 \%$ and $97.9 \pm 1.1 \%$ for single and multi lesion patients respectively). In all cases GAI exceeded the acceptance threshold of 95\%. Figure 2 shows an example of GAI analysis for a 2 lesions case using both Gafchromic and MatriXX.

Patients with 1 lesion were all treated with 2 full or partial arcs while patients with 2-3 lesions were treated with 2 to $6 \operatorname{arcs}(2.7 \pm 1.5)$. The total MUs employed

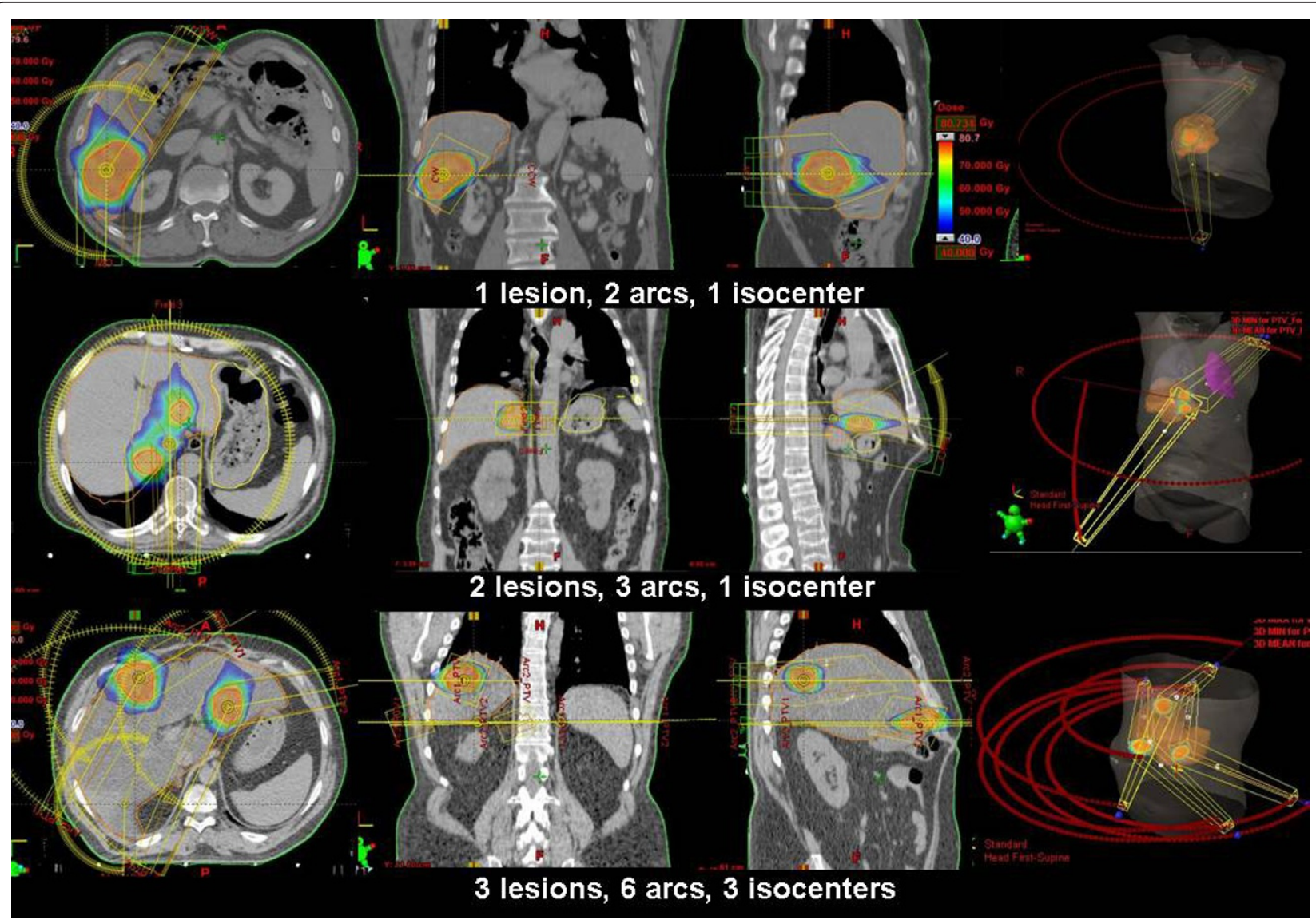

Figure 1 Examples of dose distributions. Colourwash scale is reported on the figure. Solid lines represent target volumes and organs at risk. 
Table 1 summary of dosimetric results for CTV, PTV and organs at risk

\begin{tabular}{|c|c|c|c|c|c|c|c|c|c|c|c|c|}
\hline & \multicolumn{2}{|c|}{ CTV } & \multicolumn{2}{|c|}{ PTV } & \multicolumn{2}{|c|}{ HT } & \multicolumn{2}{|c|}{ Spine } & \multicolumn{2}{|c|}{ Ipsilateral Kidney } & \multicolumn{2}{|c|}{ Liver } \\
\hline & 1 les & 2-3 les & 1 les & 2-3 les & 1 les & 2-3 les & 1 les & 2-3 les & 1 les & 2-3 les & 1 les & 2-3 les \\
\hline Vol $\left(\mathrm{cm}^{3}\right)$ & $\begin{array}{c}22.3 \pm \\
14.3\end{array}$ & $\begin{array}{c}18.6 \pm \\
15.4\end{array}$ & $\begin{array}{c}71.2 \pm \\
33.6\end{array}$ & $\begin{array}{c}72.2 \pm \\
45.8\end{array}$ & - & - & $\begin{array}{c}43.7 \pm \\
26.7\end{array}$ & $\begin{array}{c}46.0 \pm \\
19.0\end{array}$ & $\begin{array}{c}156.1 \pm \\
40.6\end{array}$ & $\begin{array}{c}154.0 \pm \\
53.4\end{array}$ & $\begin{array}{c}1349.1 \pm \\
238.4\end{array}$ & $\begin{array}{c}1708.0 \pm \\
940.7\end{array}$ \\
\hline $\begin{array}{l}\text { Mean } \\
\text { (Gy) }\end{array}$ & $\begin{array}{c}75.9 \pm \\
1.1\end{array}$ & $\begin{array}{c}75.8 \pm \\
0.6\end{array}$ & $\begin{array}{c}72.9 \pm \\
4.1\end{array}$ & $\begin{array}{c}71.5 \pm \\
4.4\end{array}$ & $\begin{array}{c}30.0 \pm \\
5.7\end{array}$ & $\begin{array}{c}30.4 \pm \\
2.6\end{array}$ & & & $3.4 \pm 4.2$ & $4.1 \pm 3.8$ & $15.1 \pm 4.4$ & $16.0 \pm 6.9$ \\
\hline $\begin{array}{l}D_{0.1} c c \\
\text { (Gy) }\end{array}$ & - & - & - & - & - & - & $9.8 \pm 4.6$ & $\begin{array}{c}12.3 \pm \\
4.2\end{array}$ & - & - & - & - \\
\hline$D_{1 \%}(G y)$ & $\begin{array}{c}77.8 \pm \\
1.3\end{array}$ & $\begin{array}{c}77.9 \pm \\
1.0\end{array}$ & $\begin{array}{c}77.4 \pm \\
1.6\end{array}$ & $\begin{array}{c}77.4 \pm \\
1.1\end{array}$ & - & - & - & - & - & - & - & - \\
\hline$D_{95 \%}(G y)$ & $\begin{array}{c}73.7 \pm \\
1.4\end{array}$ & $\begin{array}{c}74.0 \pm \\
0.8\end{array}$ & $\begin{array}{l}66.7 \pm \\
9.6\end{array}$ & $\begin{array}{c}64.7 \pm \\
9.8\end{array}$ & - & - & - & - & - & - & - & - \\
\hline$V_{10 \text { Gy }}(\%)$ & - & - & - & - & $\begin{array}{c}87.2 \pm \\
17.1\end{array}$ & $\begin{array}{c}85.3 \pm \\
3.6\end{array}$ & - & - & - & - & - & - \\
\hline $\begin{array}{l}V_{15} \mathrm{~Gy} \\
\left(\mathrm{~cm}^{3}\right)^{-}\end{array}$ & - & - & - & - & - & - & - & - & $\begin{array}{c}10.0 \pm \\
15.7\end{array}$ & $7.2 \pm 8.9$ & $\begin{array}{c}384.2 \pm \\
194.2\end{array}$ & $\begin{array}{c}425.3 \pm \\
234.2\end{array}$ \\
\hline $\mathrm{V}_{67 \%}(\%)$ & - & - & $\begin{array}{c}99.3 \pm \\
2.3\end{array}$ & $\begin{array}{c}99.8 \pm \\
0.3\end{array}$ & - & - & - & - & - & - & - & - \\
\hline $\mathrm{V}_{80 \%}(\%)$ & - & - & $\begin{array}{c}92.8 \pm \\
11.9\end{array}$ & $\begin{array}{c}89.0 \pm \\
14.2\end{array}$ & - & - & - & - & - & - & - & - \\
\hline$V_{95 \%}(\%)$ & $\begin{array}{c}99.4 \pm \\
1.56\end{array}$ & $\begin{array}{l}99.5 \pm \\
0.45\end{array}$ & $\begin{array}{c}81.6 \pm \\
26.9\end{array}$ & $\begin{array}{c}73.0 \pm \\
32.4\end{array}$ & - & - & - & - & - & - & - & - \\
\hline$V_{107 \%}(\%)$ & $0.0 \pm 0.0$ & $0.0 \pm 0.0$ & $0.1 \pm 0.2$ & $0.0 \pm 0.0$ & - & - & - & - & - & - & - & - \\
\hline $\mathrm{Cl}_{95 \%}$ & - & - & $1.1 \pm 0.2$ & $1.3 \pm 0.5$ & - & - & - & - & - & - & - & - \\
\hline
\end{tabular}

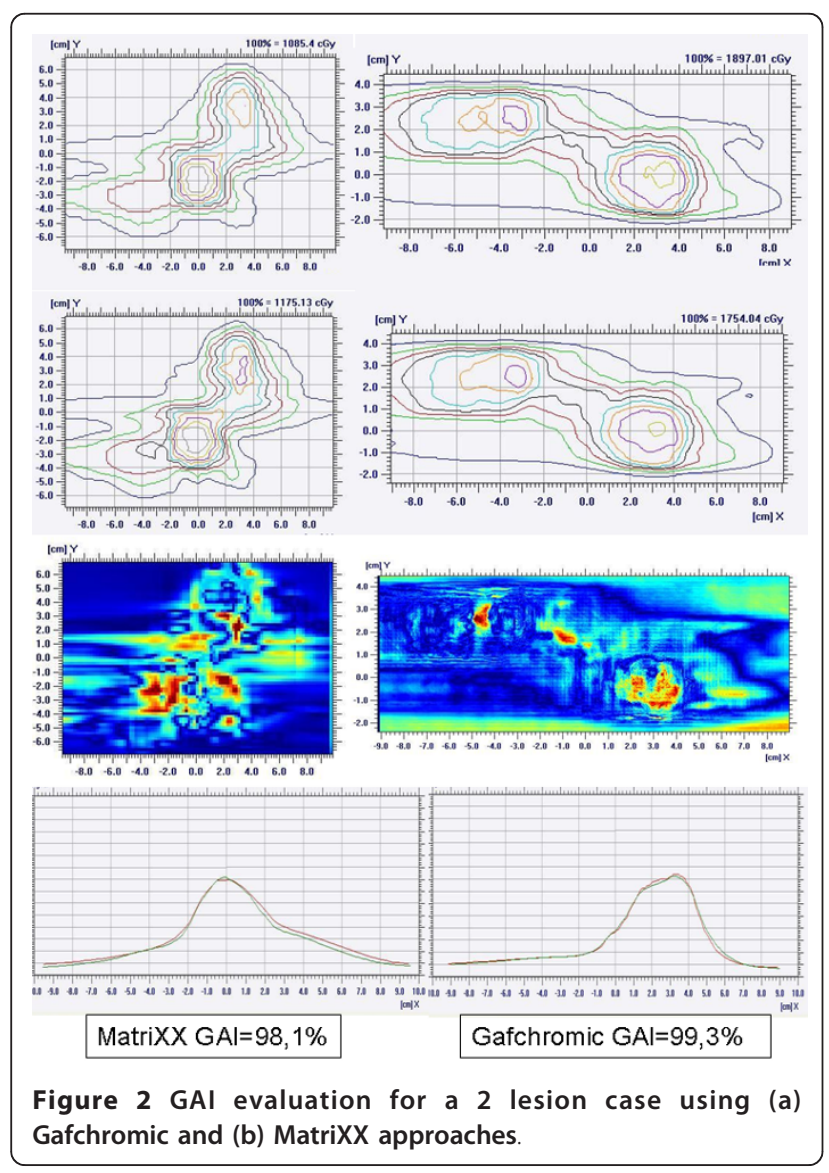

were $5241 \pm 1238$ for single lesion plans and $7550 \pm$ 3594 for the others, resulting in beam on time respectively of $2.3 \pm 0.5 \mathrm{~min}$ and $3.2 \pm 1.5 \mathrm{~min}$, with global range of 1.9-6.2 min (this last case was a three lesion with six arcs plan). The overall treatment time, including positioning, imaging, repositioning, and delivery time, was $16.2 \pm 1.7 \mathrm{~min}$ for the first fraction, lowering to $12.4 \pm 1.5 \mathrm{~min}$ for the subsequent ones. Average MU/Gy resulted in the order of 200-250, slightly increasing for multi-lesion patients $(\approx 300)$, confirming

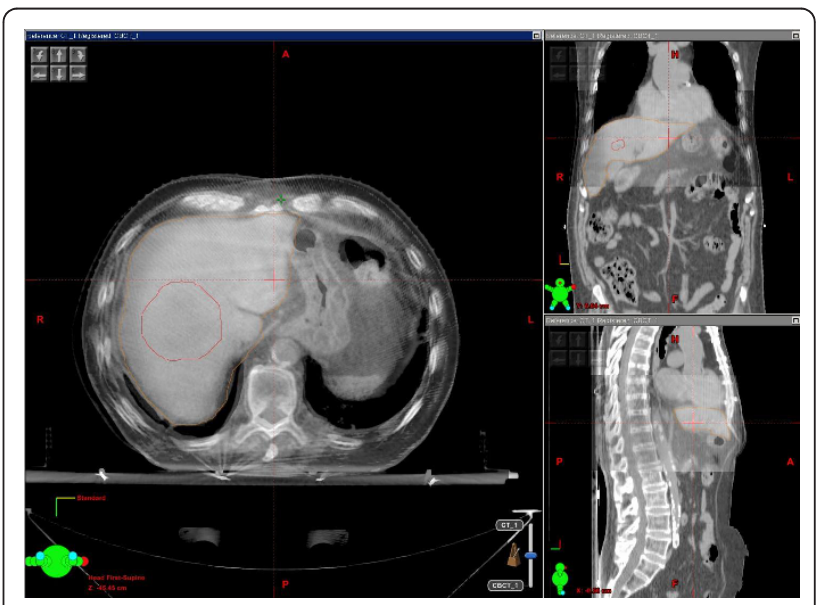

Figure 3 Daily $C B C T$ matching with the simulation $\mathrm{CT}$ for the repositioning. 
the intrinsic efficiency of RA technique also in terms of machine output factor.

Figure 3 shows a CBCT matching with the simulation CT for a patient with a single liver lesion. An analysis of the corrections applied by means of a daily CBCT was performed including all the $219 \mathrm{CBCT}$ acquired for these patients, in order to assess any systematic error due to an inaccurate patient positioning. The mean displacements found were $-0.08,0.05$ and $-0.02 \mathrm{~cm}$ with standard deviations (SD) of $0.33,0.39$ and $0.55 \mathrm{~cm}$ in vertical, longitudinal and lateral directions respectively. The random $(\sigma)$ and systematic $(\Sigma)$ population errors, as defined in [30] were calculated with the following results: $\sigma=0.25-0.26-0.34 \mathrm{~cm}, \Sigma=0.20-0.25-0.46 \mathrm{~cm}$.

\section{Discussion and Conclusions}

The use of SBRT for the irradiation of primary and metastatic tumours in several anatomical sites is becoming a standard of treatment. SBRT has emerged as a possible non invasive approach for local ablation in some series of selected cancer patients. In particular, in abdominal sites SBRT for patients with either organconfined primary tumours or oligo-metastatic disease may play a major role for improving survival in a clinically significant subset of cancer patients. Although in most cases the radiation sterilization of a metastatic lesion is not expected to lead to definitive cure, it could be effective in delaying further chemotherapy or at least this may contribute to better quality of life and local control.

The high dose per fraction of SBRT induces a huge amount of $\mathrm{MU}$ is required to cover the target and sparing the neighbour organs at risk, with a consequent increase of the beam on time with respect to the standard RT of 2 Gy/day. In this context, the recent introduction of volumetric modulated arc therapy, e.g. RapidArc, has shown to reduce the total monitor units and thus the treatment time in comparison with intensity modulated RT (IMRT) without compromising the target coverage as shown in many anatomical regions.

In this paper we considered the first 55 consecutive patients that underwent SBRT on both primary and metastatic hepatic targets, using FFF beams. In particular 43 patients presented a single lesion, 9 two lesions and 3 presented three lesions. Removal of the flattening filter gives the possibility to deliver treatments with higher dose rates, up to a factor 4 at $10 \mathrm{MV}$, and with a much higher dose per pulse. The different physical characteristics of the FFF beams (e.g. the different beam profile) are under investigation by the scientific community [18-23] and first preliminary clinical data are in progress [25].

In a previous report we analyzed a cohort of patients treated with RapidArc technique for abdominal lesions, including abdominal lymph-nodes, pancreas, and liver metastases [16]. All plans were optimized using flattened beams. In particular, comparing the results obtained for the liver metastases cohort in [16] and the data reported in this study it appears that, though for all patients $95 \%$ of the PTV volume received at least $67 \%$ of the prescription dose, more patients could receive higher mean doses to the PTV with FFF beams: mean dose to PTV was $67 \mathrm{~Gy}$ in [16] and around 73 Gy in the present study, PTV V95\% passed from $40 \%$ to $81 \%$, of course the higher doses to the target implies higher doses to OARs (e.g. liver V15Gy passed from 257 to $384 \mathrm{cc}$ ) but the constraints were always respected, the mean GAI value improved passing from $97.8 \%$ to $98.7 \%$. On this topic, in a recent paper, we specifically compared FFF and FF beams from a dosimetric prospective, demonstrating by a theorical point of view FFF beam to be adequate in abdominal SBRT for lesions from small to medium sizes (i.e. up to 200-300 cc), with adeguate healthy tissue sparing and PTV coverage with respect to flattened beams [32]. The only significant variation between the two cohorts was the beam on time. In the previous study, where only single hepatic metastases were considered, around 9 minutes were necessary to deliver the treatment while in the present group of patients the beam on time was reduced to around 2.3 minutes (considering only the single-lesion patients), with a cut of time of more than $350 \%$, decreasing patient's discomfort and reducing intra-fraction uncertainties. In both series the instantaneous delivery dose rate was almost always at the maximum value, $600 \mathrm{MU} /$ min for FF beams and $2400 \mathrm{MU} / \mathrm{min}$ for FFF, hence the beam on time reduction. Furthermore the data reported in the present study showed that plans for with multilesion patients are dosimetrically comparable with the single lesion ones.

In our Institution, image-guidance by means of CBCT, implemented in the therapeutic radiation device, is daily used to better define and correct setup of each patient before each fraction of the treatment. The analysis of the daily displacements, in particular the mean values very close to 0 , show the efficiency of our set-up method; however, the calculation of $\Sigma$ and $\sigma$ and of the relative margins, shows the absolute necessity of daily repositioning by means of $\mathrm{CBCT}$; the efficiency of the set-up alone with abdominal compression and stereotactic body frame is not sufficient to apply margins of 5-8 $\mathrm{mm}$ in the anterior-posterior and lateral axes. Daily Image guidance has therefore allowed the minimizing of the set-up margins from CTV to PTV, reducing the normal tissue surrounding the target close to healthy organs in critical sites, such as in abdomen. At this purpose, Eccles et al. from Princess Margaret Hospital showed, in case of daily CBCT linked with abdominal 
compression, the maximum changes in tumour center of mass to be lower than $5 \mathrm{~mm}$ in $94 \%$ patients, with mean displacement of $1.4 \mathrm{~mm}, 2.1$, and $1.0 \mathrm{~mm}$ in, respectively, LR, AP, CC directions [25].

In conclusion, we reported our practice in the treatment of liver metastases on the first 55 patients using FFF beams, prescribing 75 Gy in 3 fractions at PTV. In the majority of cases the beam on time was lower than 3 minutes, strongly reducing the treatment time in comparison with flattened filter beams, without compromising the target coverage and organs at risk sparing.

\section{Author details}

${ }^{1}$ IRCCS Istituto Clinico Humanitas, Rozzano (Milano), Italy. ${ }^{2}$ Oncology Institute of Southern Switzerland, Bellinzona, Switzerland.

\section{Authors' contributions}

GR, MC, CP, SA, FL, AT, AF and LC carried out the data and participated in the data evaluation. PM, SC and GR drafted the manuscript. PM, SC and GR participated in the design of the study. FA, AF, PN and LC performed the statistical analysis. SC, FA, SA, AT, PN and MS carried out the patients record evaluation and followed patients and treatments. The definitive supervision of the paper was done by PM and MS. All authors read and approved the final manuscript.

\section{Competing interests}

Dr. L. Cozzi is Head of Research at Oncology Institute of Southern Switzerland and acts as a Scientific Advisor to Varian Medical Systems. Other authors have no conflict of interest.

\section{Received: 30 December 2011 Accepted: 1 February 2012}

Published: 1 February 2012

\section{References}

1. Timmerman RD, Kavanagh BD, Cho LC, Papiez L, Xing L: Stereotactic body radiation therapy in multiple organ sites. $J$ Clin Oncol 2007, 25:947-952.

2. Macdermed DM, Weichselbaum RR, Salama JK: A rationale for the targeted treatment of oligometastases with radiotherapy. J Surg Oncol 2008, 98:202-206.

3. Schefter $T E$, Kavanagh $B D$, Timmerman RD, Cardenes $H R$, Baron $A$, Gaspar LE: A phase I trial of stereotactic body radiation therapy (SBRT) for liver metastases. Int J Radiation Oncol Biol Phys 2005, 62:1371-1378.

4. Katz AW, Carey-Sampson M, Muhs AG, Milano MT, Schell MC, Okunieff P: Hypofractionated stereotactic body radiation therapy (SBRT) for limited hepatic metastases. Int J Radiation Oncology Biol Phys 2007, 67:793-798.

5. Scorsetti M, Fogliata A, Castiglioni S, et al: Early clinical experience with volumetric modulated arc therapy in head and neck cancer patients. Radiation Oncology 2010, 5:93.

6. Scorsetti M, Navarria P, Alongi F, et al: Large volume unresectable locally advanced non-small cell lung cancer: acute toxicity and initial outcome results with rapid arc. Radiation Oncology 2010, 5:94

7. Scorsetti M, Bignardi $M$, Clivio A, et al: Volumetric modulation arc radiotherapy compared with static gantry intensity-modulated radiotherapy for malignant pleural mesothelioma tumor: a feasibility study. Int J Radiat Oncol Biol Phys 2010, 77:942-949.

8. Fogliata A, Bergstrom S, Cafaro I, et al: Cranio-spinal irradiation with volumetric modulated arc therapy: a multi-institutional treatment experience. Radiotherapy and Oncology 2011, 99:79-85.

9. Mancosu P, Cozzi L, Fogliata A, et al: Collimator angle influence on dose distribution optimization for vertebral metastases using volumetric modulated arc therapy. Med Phys 2010, 37:4133-4137.

10. Mancosu P, Navarria P, Bignardi M, et al: Re-irradiation of metastatic spinal cord compression: a feasibility study by volumetric-modulated arc radiotherapy for in-field recurrence creating a dosimetric hole on the central canal. Radiotherapy and Oncology 2010, 94:67-70.

11. Fogliata A, Cozzi L, Clivio A, et al: Preclinical assessment of volumetric modulated arc therapy for total marrow irradiation. Int J Radiat Oncol Biol Phys 2011, 80:628-636.

12. Mancosu P, Navarria P, Castagna L, et al: Anatomy driven optimisation strategy for total marrow irradiation with a volumetric modulated arc therapy technique. JACMP 2011.

13. Scorsetti M, Mancosu $P$, Navarria $P$, et al: Stereotactic body radiation therapy (SBRT) for adrenal metastases: a feasibility study of advanced techniques with modulated photons and protons. Strahlenther Onkol 2011, 187:238-244

14. Bignardi M, Cozzi L, Fogliata A, Lattuada P, Mancosu P, Navarria P, et al: Critical appraisal of volumetric modulated arc therapy in stereotactic body radiation therapy for metastases to abdominal lymph nodes. Int J Radiat Oncol Biol Phys 2009, 75:1570-1577.

15. Bignardi M, Navarria P, Mancosu P, Cozzi L, Fogliata A, Tozzi A, et al: Clinical outcome of hypofractionated stereotactic radiotherapy for abdominal lymph node metastases. Int J Radiati Oncol Biol Phys Vol 2010.

16. Scorsetti M, Bignardi M, ALongi F, Fogliata A, Mancosu P, Navarria P Castiglioni S, Pentimalli S, Tozzi A, Cozzi L: Stereotactic body radiation therapy for abdominal targets using volumetric intensity modulated arc therapy with RapidArc: feasibility and clinical preliminary results. Acto Oncol 2011, 50(4):528-38.

17. Hrbacek J, Lang S, Klöck S: Comissioning of photon beams of a flattening filter-free linear accelerator and the accuracy of beam modelling using an anisotropic analytical algorithm. Int I Radiat Oncol Biol Phys .

18. Vassiliev ON, Titt U, Pönisch F, Kry SF, Mohan R, Gillin MT: Dosimetric properties of photon beams from a flattening filter free clinical accelerator. Phys Med Biol 2006, 51:1907-17.

19. Kry SF, Howell RM, Titt U, Salehpour M, Mohan R, Vassiliev ON: Energy spectra, sources, and shielding considerations for neutrons generated by a flattening filter-free Clinac. Med Phys 2008, 35(5):1906-11.

20. Pönisch F, Titt U, Vassiliev ON, Kry SF, Mohan R: Properties of unflattened photon beams shaped by a multileaf collimator. Med Phys 2006, 33(6):1738-46.

21. Vassiliev ON, Titt U, Kry SF, Pönisch F, Gillin MT, Mohan R: Monte Carlo study of photon fields from a flattening filter-free clinical accelerator. Med Phys 2006, 33(4):820-7.

22. Georg D, Knöös T, McClean B: Current status and future perspective of flattening filter free photon beams. Med Phys 2011, 38(3):1280-93.

23. Kragl G, Baier F, Lutz S, Albrich D, Dalaryd M, Kroupa B, Wiezorek T, Knöös T, Georg D: Flattening filter free beams in SBRT and IMRT: dosimetric assessment of peripheral doses. Z Med Phys 2010

24. Vassiliev ON, Kry SF, Chang JY, Balter PA, Titt U, Mohan R: Stereotactic radiotherapy for lung cancer using a flattening filter free Clinac. J Appl Clin Med Phys 2009, 10(1):2880..

25. Scorsetti M, Alongi F, Castiglioni S, Clivio A, Fogliata A, Lobefalo F, Mancosu P, Navarria P, Palumbo V, Pellegrini C, Pentimalli S, Reggiori G, Ascolese AM, Roggio A, Arcangeli S, Tozzi A, Vanetti E, Cozzi L: Feasibility and early clinical assessment of flattening filter free (FFF) based stereotactic body radiotherapy (SBRT) treatments. Radiat Oncol 2011, 6:113

26. Eccles CL, Dawson L, Moseley JL, Brock KK: Intrafraction liver shape variability and impact on GTV position during liver stereotactic radiotherapy using abdominal compression. Int I Radiat Oncol Biol Phys 2011, 80(3):938-946.

27. Reggiori G, Mancosu P, Tozzi A, et al: Cone beam CT pre- and post-daily treatment for assessing geometrical and dosimetric intrafraction variability during radiotherapy of prostate cancer. J Appl Clin Med Phys 2010, 12:3371.

28. RAS-trial radiofrequency ablation versus stereotactic body radiation therapy for colorectal liver metastases: a randomized trial. , Available at: http://www.livertumor.dk/RAS-trial.html.

29. Milano MT, Constine LS, Okunieff P: Normal tissue toxicity after small field hypofractionated stereotactic body radiation. Radiation Oncology 2008, 3:36. 
30. Van Herk M, Remeijer P, Rasch C, Lebesque JV: The probability of correct target dosage: dose-population histograms for deriving treatment margins in radiotherapy. Int J Radiat Oncol Biol Phys 2000, 47(4):1121-35.

31. Low DA, Harms WB, Mutic S, Purdy JA: A technique for quantitative evaluation of dose distributions. Med Phys 1998, 25:656-661.

32. Reggiori G, Mancosu P, Castiglioni $S$, et al: Can volumetric modulated arc therapy with flattening filter free beams play a role in stereotactic body radiotherapy for liver lesions? Med Phys 2012.

doi:10.1186/1748-717X-7-16

Cite this article as: Mancosu et al: Stereotactic body radiation therapy for liver tumours using flattening filter free beam: dosimetric and technical considerations. Radiation Oncology 2012 7:16.

Submit your next manuscript to BioMed Central and take full advantage of:

- Convenient online submission

- Thorough peer review

- No space constraints or color figure charges

- Immediate publication on acceptance

- Inclusion in PubMed, CAS, Scopus and Google Scholar

- Research which is freely available for redistribution

Submit your manuscript at www.biomedcentral.com/submit 